\title{
ARTICLE \\ Perinatal THC exposure via lactation induces lasting alterations to social behavior and prefrontal cortex function in rats at adulthood
}

\author{
Andrew F. Scheyer $\mathbb{D}^{1,2,3}$, Milene Borsoi ${ }^{1,2,3}$, Anne-Laure Pelissier- Alicot ${ }^{1,2,3,4,5}$ and Olivier J. J. Manzoni $\mathbb{D}^{1,2,3}$
}

\begin{abstract}
Cannabis is the world's most widely abused illicit drug and consumption amongst women during and surrounding the period of pregnancy is increasing. Previously, we have shown that cannabinoid exposure via lactation during the early postnatal period disrupts early developmental trajectories of prefrontal cortex maturation and induces behavioral abnormalities during the first weeks of life in male and female rat progeny. Here, we investigated the lasting consequences of this postnatal cannabinoid exposure on synaptic and behavioral parameters in the adult offspring of $\Delta$-tetrahydrocannabinol (THC)-treated dams. At adulthood, these perinatally THC-exposed rats exhibits deficits in social discrimination accompanied by an overall augmentation of social exploratory behavior. These behavioral alterations were further correlated with multiple abnormalities in synaptic plasticity in the prefrontal cortex, including lost endocannabinoid-mediated long-term depression (LTD), lost long-term potentiation and augmented mGlu2/3-LTD. Finally, basic parameters of intrinsic excitability at prefrontal cortex pyramidal neurons were similarly altered by the perinatal THC exposure. Thus, perinatal THC exposure via lactation induces lasting deficits in behavior and synaptic function which persist into adulthood life in male and female progeny.
\end{abstract}

Neuropsychopharmacology (2020) 45:1826-1833; https://doi.org/10.1038/s41386-020-0716-x

\section{INTRODUCTION}

Cannabis is currently the most widely used illicit drug in the world, and its consumption by pregnant women has been steadily increasing [1-4]. Further, the majority of pregnant women estimate little-or-no risk associated with cannabis use during pregnancy [5], and a trend towards recommendations for cannabis use during the perinatal period has been noted in areas of the United States where cannabis use has been legalized [6]. During the early postnatal period, breastfeeding by cannabisusing mothers actively transfers the principle psychoactive component of cannabis, $\Delta 9$-tetrahydrocannabinol (THC) and other cannabinoids to the developing infant, as the lipophilic nature of these cannabinoids results in concentrations in breast milk which are equal to or exceeding that found in the plasma of the user $[7,8]$. Importantly, this transfer may persist well beyond the intoxication period of the user due to the long half-life of THC in breast milk [9], thus occluding the possibility of simply breastfeeding outside of the acute cannabis consumption period as is often done with alcohol consumption [10]. Therefore, understanding and disseminating data regarding the consequences of THC consumption during the breastfeeding period is paramount to public health in the evolving age of cannabis use.

During prenatal and early postnatal development, herein referred to as the perinatal period [11, 12], the developing brain is acutely sensitive to exogenous cannabinoids. Indeed, the endocannabinoid system (ECS), comprised of the cannabinoid receptors (CB1R and $C B 2 R)$, as well as the endogenous cannabinoids anandamide (AEA) and 2-arachidonoylglycerol (2-AG) amongst other elements, is intimately involved in development beginning at ontogenesis $[13,14]$ and continuing throughout the neurodevelopmental period [15-19]. Thus, it is not surprising that in utero exposure to THC induces wide-ranging, multi-scale lasting effects in a variety of brain regions and behavioral domains.

One such impacted brain region is the prefrontal cortex (PFC), a functional hub which plays a central role in such diverse cognitive realms as working memory, reasoning, cognitive flexibility and emotionally-guided behaviors including social interactions $[20,21]$. The PFC is also a site of dense expression of CB1R $[21,22]$ and is exquisitely sensitive to various ECS-related synaptopathies which manifest as a variety of cognitive disease states [23]. Previously, our lab has demonstrated that exogenous cannabinoid exposure during in utero development results in significant abnormalities in PFC synaptic function which underlie behavioral alterations lasting into adulthood in cannabinoidexposed progeny $[24,25]$.

During the early postnatal period, we have shown that exposure to exogenous cannabinoids via lactation also alters the developmental trajectory of the PFC $[26,27]$. Specifically, the timeline of cell excitability changes mediated by GABAergic maturation is delayed by exposure to either THC or a synthetic agonist of CB1R. This delayed PFC development is accompanied by select behavioral abnormalities which similarly indicate a retarded

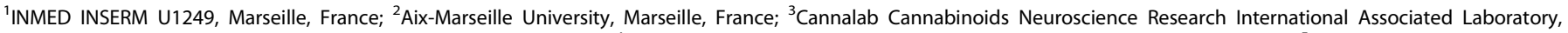

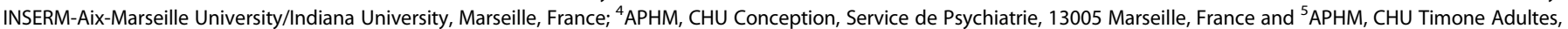
Service de Médecine Légale, 13005 Marseille, France

Correspondence: Olivier J. J. Manzoni (olivier.manzoni@inserm.fr)

Received: 3 February 2020 Revised: 16 April 2020 Accepted: 13 May 2020

Published online: 19 May 2020 
maturational profile in the cannabinoid-exposed offspring. Here, we extend upon these findings by investigating the long-term consequences of this perinatal THC exposure. We observed that, at adulthood ( $>90$ postnatal days; PND), the offspring of dams treated with THC from PND1-10 exhibit augmented social behavior at the cost of social memory discrimination, as well as altered multi-directional synaptic plasticity and cell-excitability in the PFC. These data illuminate significant consequences resultant of perinatal THC exposure via lactation which may serve to inform breastfeeding mothers during the early postnatal period and provide fundamental bases for strategic interventions to correct such effects in impacted progeny.

\section{MATERIALS AND METHODS}

Further information and requests for resources/reagents should be directed to the Lead Contact, Olivier J.J. Manzoni (olivier. manzoni@inserm.fr).

\section{Animals}

Animals were treated in compliance with the European Communities Council Directive (86/609/EEC) and the United States NIH Guide for the Care and Use of Laboratory Animals. The French Ethical committee authorized the project "Exposition Périnatale aux cannabimimétiques" (APAFIS\#18476-2019022510121076 v3). All rats were group-housed with $12 \mathrm{~h}$ light/dark cycles with ad libitum access to food and water. All behavioral, biochemical and synaptic plasticity experiments were performed on male and female RjHan:wi-Wistar rats (>P90) from pregnant females obtained from Janvier Labs. Pregnant dams arrived at E15 and remained undisturbed until delivery. Newborn litters found before 05:00p.m. were considered to be born that day (P0).

Dams were injected daily sub-cutaneously (s.c.) from P01-10 with $\Delta$ 9-Tetrahydrocannabinol (THC; $2 \mathrm{mg} / \mathrm{kg} /$ day). THC was suspended in 1:1:18 DMSO, Cremophor and saline, and injected at $1 \mathrm{ml} / \mathrm{kg}$. Control dams (Sham) received vehicle.

All groups (both electrophysiology and behavior) represent data from a minimum of 2 litters. Animals used for behavioral analyses were also used for electrophysiological data acquisition in order to minimize the total number of animals required for the experiments, as requested by ethical directives. Female data were collected blind to the estrous cycle. All datasets are collapsed amongst male/female rats. Sex-specific data for each figure can be found in a corresponding supplementary table.

\section{Behavior}

Open field observations were conducted after rats were adapted to the room laboratory conditions for at least $1 \mathrm{~h}$ prior to testing. Tests were conducted in a $45 \times 45 \mathrm{~cm}$ Plexiglass arena with $\pm 2 \mathrm{~cm}$ of wood shavings covering the floor. All behavioral procedures were performed between 10:00 am and 3:00 pm. All sessions were recorded using a video camera using the Ethovision XT 13.0 video tracking software (Noldus, Netherlands) and analyzed by a trained observer who was unaware of treatment condition.

The social interaction apparatus consisted of a transparent acrylic chamber $(120 \times 80 \mathrm{~cm})$ divided into three equal compartments $(40 \mathrm{~cm}$ each) partially separated by white walls. The central compartment was empty and lateral compartments had an empty wire cage $(20 \mathrm{~cm}$ diameter) were an object or a new rat (social stimulus) were placed during the test.

THC- or sham-exposed rats were individually habituated to the test cage containing the two empty wire cages for $10 \mathrm{~min}$ immediately prior to testing. The first trial (social approach, $5 \mathrm{~min}$ duration) consisted of giving the tested rat the option to socialize with either a novel object or a new, naïve, age- and sex-mate conspecific rat that were placed into the wire cages positioned on the arena's opposite sides. Thirty minutes later, the tested rat returned to the apparatus for the second trial (social memory,
5 min duration) wherein the two compartments held either the now-familiar rat from the first testing phase or a second, previously unknown, naïve, age- and sex-mate conspecific.

Only rats with no compartment preference during the habituation phase were used.

Time spent in each compartment and time spent exploring wire cages during the social approach and social memory phases were scored. Social Preference Ratio was calculated as time spent exploring either the wire cage containing the object, or the new rat divided by total time exploring both wire cages. Likewise, Social Memory Ratio was calculated as time spent exploring either the wire cage containing the rat used in the first trial or the new rat divided by total time exploring both wire cages.

Slice preparation

Adult male and female rats were anesthetized with isoflurane and killed as previously described $[25,26,28,29]$. The brain was sliced $(300 \mu \mathrm{m})$ in the coronal plane with a vibratome (Integraslice, Campden Instruments) in a sucrose-based solution at $4{ }^{\circ} \mathrm{C}$ (in $\mathrm{mm}$ as follows: $87 \mathrm{NaCl}, 75$ sucrose, 25 glucose, $2.5 \mathrm{KCl}, 4 \mathrm{MgCl}_{2}, 0.5$ $\mathrm{CaCl}_{2}, 23 \mathrm{NaHCO}_{3}$ and $1.25 \mathrm{NaH}_{2} \mathrm{PO}_{4}$ ). Immediately after cutting, slices containing the medial prefrontal cortex (PFC) were stored for $1 \mathrm{~h}$ at $32{ }^{\circ} \mathrm{C}$ in a low-calcium ACSF that contained (in $\mathrm{mm}$ ) as follows: $130 \mathrm{NaCl}, 11$ glucose, $2.5 \mathrm{KCl}, 2.4 \mathrm{MgCl}_{2}, 1.2 \mathrm{CaCl}_{2}, 23$ $\mathrm{NaHCO}_{3}, 1.2 \mathrm{NaH}_{2} \mathrm{PO}_{4}$, and were equilibrated with $95 \% \mathrm{O}_{2} / 5 \% \mathrm{CO}_{2}$ and then at room temperature until the time of recording. During the recording, slices were placed in the recording chamber and superfused at $2 \mathrm{ml} / \mathrm{min}$ with low $\mathrm{Ca}^{2+}$ ACSF. All experiments were done at $32^{\circ} \mathrm{C}$. The superfusion medium contained picrotoxin (100 mM) to block gamma-aminobutyric acid types A (GABA-A) receptors. All drugs were added at the final concentration to the superfusion medium.

\section{Electrophysiology}

Whole-cell patch-clamp of visualized layer five pyramidal neurons mPFC and field potential recordings were made in coronal slices containing the mPFC as previously described [26, 27, 29, 30]. Neurons were visualized using an upright microscope with infrared illumination. The intracellular solution was based on $\mathrm{K}+$ gluconate (in $\mathrm{mM}: 145 \mathrm{~K}$ + gluconate, $3 \mathrm{NaCl}, 1 \mathrm{MgCl}_{2}, 1 \mathrm{EGTA}$, $0.3 \mathrm{CaCl}_{2}, 2 \mathrm{Na}^{2+}$ ATP, and $0.3 \mathrm{Na}^{+} \mathrm{GTP}, 0.2$ cAMP, buffered with 10 HEPES). To quantify the AMPA/NMDA ratio we used a $\mathrm{CH}_{3} \mathrm{O}_{3} \mathrm{SCs}$-based solution (in mM: $128 \mathrm{CH}_{3} \mathrm{O}_{3} \mathrm{SCs}, 20 \mathrm{NaCl}, 1 \mathrm{MgCl}_{2}$, 1 EGTA, $0.3 \mathrm{CaCl}_{2}, 2 \mathrm{Na}^{2+}$ ATP, and $0.3 \mathrm{Na}^{+}$GTP, $0.2 \mathrm{cAMP}$, buffered with 10 HEPES, pH 7.2, osmolarity 290-300 mOsm). The $\mathrm{pH}$ was adjusted to 7.2 and osmolarity to $290-300 \mathrm{mOsm}$. Electrode resistance was 4-6 MOhms.

Whole-cell patch-clamp recordings were performed with an Axopatch-200B amplifier as previously described [31]. Data were low pass filtered at $2 \mathrm{kHz}$, digitized $(10 \mathrm{kHz}$, DigiData $1440 \mathrm{~A}$, Axon Instrument), collected using Clampex 10.2 and analyzed using Clampfit 10.2 (all from Molecular Device, Sunnyvale, USA).

A $-2 \mathrm{mV}$ hyperpolarizing pulse was applied before each evoked EPSC in order to evaluate the access resistance and those experiments in which this parameter changed $>25 \%$ were rejected. Access resistance compensation was not used, and acceptable access resistance was $<30 \mathrm{MOhms}$. The potential reference of the amplifier was adjusted to zero prior to breaking into the cell. Cells were held at $-75 \mathrm{mV}$.

Current-voltage (I-V) curves were made by a series of hyperpolarizing to depolarizing current steps immediately after breaking into the cell. Membrane resistance was estimated from the I-V curve around resting membrane potential [11, 26, 31, 32].

Field potential recordings were made in coronal slices containing the mPFC or the accumbens as previously described [29]. During the recording, slices were placed in the recording chamber and superfused at $2 \mathrm{ml} / \mathrm{min}$ with low $\mathrm{Ca}^{2+}$ ACSF. All experiments were done at $32^{\circ} \mathrm{C}$. The superfusion medium contained picrotoxin 
(100 mM) to block GABA Type A (GABA-A) receptors. All drugs were added at the final concentration to the superfusion medium. The glutamatergic nature of the field EPSP (fEPSP) was systematically confirmed at the end of the experiments using the ionotropic glutamate receptor antagonist CNQX $(20 \mathrm{mM})$, which specifically blocked the synaptic component without altering the non-synaptic.

Both fEPSP area and amplitude were analyzed. Stimulation was performed with a glass electrode filled with ACSF and the stimulus intensity was adjusted $\sim 60 \%$ of maximal intensity after performing an input-output curve (baseline EPSC amplitudes ranged between 50 and $150 \mathrm{pA}$ ). Stimulation frequency was set at $0.1 \mathrm{~Hz}$.

Data acquisition and analysis

The magnitude of plasticity was calculated at $0-10 \mathrm{~min}$ and $35-40$ min after induction (for TBS-LTP and eCB-LTD) or drug application (mGlu2/3-LTD) as percentage of baseline responses. Statistical analysis of data was performed with Prism (GraphPad Software) using tests indicated in the main text after outlier subtraction (Grubb's test, alpha level 0.05). All values are given as mean \pm SEM, and statistical significance was set at $p<0.05$.

\section{RESULTS}

Perinatal THC exposure augments social exploration at the cost of discrimination between novel and familiar social stimuli

In rodent models, exposure to cannabinoids (both synthetic and plant-derived) during gestation or early development induces an array of deleterious consequences on behavior manifesting both at early life and adulthood [31]. Previously, we have shown that the administration of cannabinoids to lactating dams during the early postnatal period causes significant delays in both synaptic and behavioral development [20]. Here, we used the same protocol of perinatal cannabinoid exposure in order to determine if such earlylife exposure results in consequences lasting into adulthood. Thus, lactating rat dams were treated with a single daily low dose of $\Delta 9$ Tetrahydrocannabinol (THC; $2 \mathrm{mg} / \mathrm{kg}$, S.C.) or its vehicle (herein referred to as "sham") from postnatal day (PND) 1-10. Experiments were conducted in both sexes of their adult offspring (>PND90).

First, in order to determine if simple parameters of naturalistic behavior were altered in the adult offspring of dams exposed to THC during the early postnatal period, we tested these animals in the open field environment (Fig. 1a-c). Here, we did not observe any significant differences between the THC-exposed animals and those originating from sham-treated dams. Specifically, no differences were found in the time spent in the center of the arena, the total distance covered during the test, or the number of rearing events. Thus, perinatal THC exposure via lactation does not appear to alter baseline levels of spontaneous activity or anxiety at adulthood. Sexspecific details can be found in supplementary Table 1.

Next, based on previous findings [25] from our lab which have revealed sex-specific deficits in social interaction following in utero exposure to cannabinoids, we tested the adult offspring of dams given THC during the early postnatal period in a two-stage social task to assess both social preference/approach (Fig. 1d-f) and social memory (Fig. 1g-i).

Here, we found that during the initial testing period, the adult offspring of sham- or THC-treated dams both exhibited a significant preference for exploring the novel rat rather than the novel object, as determined by time spent at the two cages containing the social stimulus (Fig. 1e). Interestingly, this preference, as determined by a ratio between the time spent at these two sites, unveiled a stronger social preference amongst THC-exposed rats, as compared to the sham group (Fig. 1d). No significant difference was noted in the total time spent exploring both cages (Fig. 1f). Therefore, both sham- and THC-exposed rats exhibit significant social preference over novel object stimuli, a behavioral characteristic which appears to be augmented in the latter group.
Next, in the social memory task, sham-exposed rats displayed the expected preference for a novel rat over the familiar rat from the first testing period (Fig. 1g-h). However, THC-exposed rats failed to exhibit such a preference, as revealed by equivalent amounts of time spent exploring the cages containing both the novel and familiar stimulus rats (Fig. 1h). Interestingly, the total time spent exploring non-discriminated rats was significantly higher in the THC-exposed rats, as compared to the sham group (Fig. 1i). This significant difference owes to increased time spent on the familiar rat, without a lower amount of time spent on the novel rat, as compared to these times of exploration exhibited by the sham-exposed rats. Thus, we conclude that perinatal THC exposure increases overall social exploration at the cost of reducing discrimination between novel and familiar social stimuli. Sexspecific details can be found in supplementary Table 2 .

Finally, animals exposed to THC via lactation were also tested in the Novel Object Recognition test. We found no differences in this test suggesting that our protocol of perinatal THC exposure does not cause massive/widespread cognitive deficits (Discrimination Index $=0.6136$ and 0.5809 for Sham and THC, respectively; $N=22$ and 23 , respectively; Two-tailed T-test, $P=0.4428$ ).

Perinatal THC exposure alters multiple forms of synaptic plasticity in the PFC at adulthood

The PFC plays a significant role in social behavior [33]. Previously, we have shown that deficits in social behavior such as those described here are correlated with alterations to synaptic plasticity in the PFC [25] including a loss of eCB-mediated long-term depression (LTD) induced by a $10-\mathrm{min}, 10 \mathrm{~Hz}$ stimulation at excitatory synapses onto layer 5 PFC pyramidal cells. Thus, we next investigated this form of LTD in the PFC of adult rats exposed to THC during the early perinatal period (Fig. 2).

First, we established that a 10 -minute, $10 \mathrm{~Hz}$ stimulation of superficial-layers of the PFC in slices obtained from the adult offspring of sham-injected dams induces a robust LTD at layer 5 synapses (Fig. 2a, b). However, this same protocol failed to elicit LTD in slices obtained from THC-exposed animals. Thus, we can conclude that perinatal THC exposure induces a lasting deficit in eCB-LTD in the PFC.

To determine if this ablation of LTD in the PFC was limited to eCB-LTD or a more global impact, we next examined a distinct form of LTD in the PFC mediated by mGlu2/3 receptors [34, 35] which has previously been shown to be disrupted by chronic exposure to drugs of abuse [36] but not by in utero cannabinoid exposure [25]. Previous work has shown that mGlu2/3 LTD and eCB-LTD can mutually occlude each other in the nucleus accumbens [37]. Further, shared pools of $G_{i / o}$, normally sequestered by CB1R [38], are likely made more available as a result of CB1R desensitization/ablation of eCB-LTD, thereby permitting enhanced mGlu2/3-LTD [37]. We therefore sought to test this possibility in our THC-exposed offspring by exposing PFC slices for 10-minutes to the mGlu2/3 agonist, LY379268 (300 nM) in order to induce an mGlu2/3-dependent LTD. As predicted, this drug application elicited a significant LTD at layer 5 synapses in slices obtained from the adult offspring of sham-treated dams (Fig. 2c, d). Similarly, the 10-minute application effectively elicited LTD in slices obtained from THC-exposed rats. Interestingly, the magnitude of this mGlu2/3 LTD is significantly larger in the THC-exposed rats, as compared to sham-exposed rats. Previously, a similar compensatory enhancement of mGlu2/3 LTD has been noted in mice chronically exposed to THC wherein eCB-LTD is abolished [37]. Thus, perinatal THC exposure appears to abolish eCB-LTD while enhancing mGlu2/3-LTD in the PFC of adult rats. Sex-specific details can be found in supplementary Table 3 .

eCB-LTD requires the participation of endocannabinoids, particularly 2-AG in the PFC [31]. We sought to determine if enhanced levels of 2AG could benefit to eCB-LTD in THC exposed rats. Thus, PFC slices were incubated in JZL184 ( $4 \mu \mathrm{M}$, a potent 
a

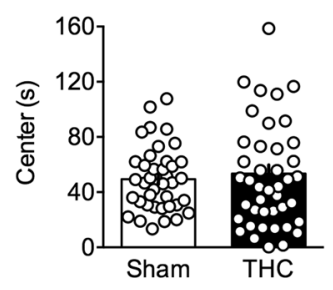

d

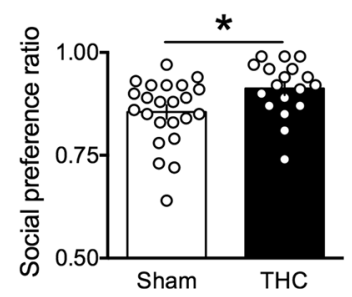

g

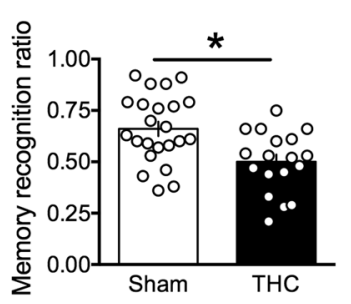

b

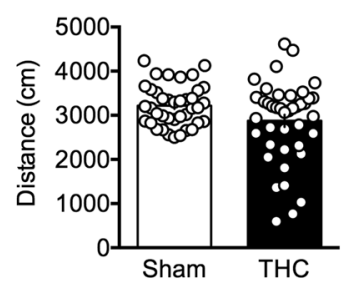

e

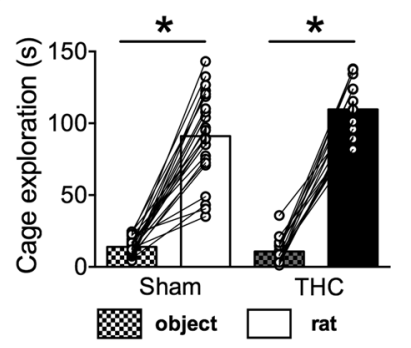

h

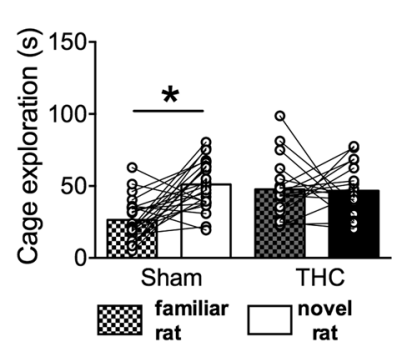

C

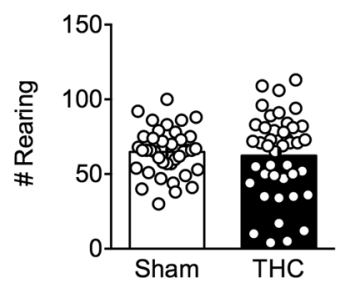

f

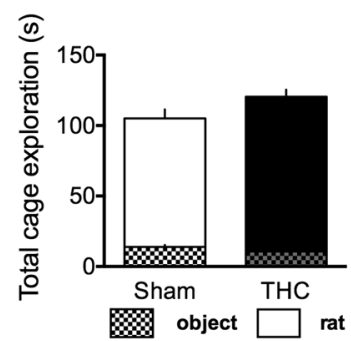

i

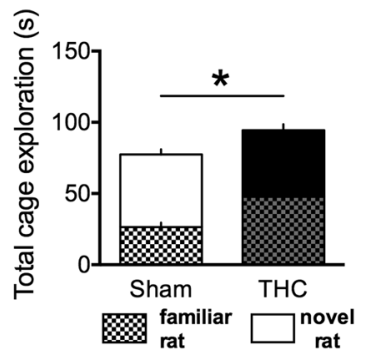

Fig. 1 Perinatal THC exposure alters social approach and memory without changing behavior in the open field environment. a-c: Behaviors in the open field arena were not altered in the adult offspring of THC-treated as compared to those from sham-treated dams $(N=42,40$ respectively). Specifically, no differences were found in the total distance covered during the trial, the bouts of rearing behavior, nor in the time spent in the center of the arena (Two-tailed T-test, $P=0.1846,0.9644$ and 0.7765 , respectively). d-f Social discrimination between an object and a novel rat is enhanced in the adult offspring of THC-treated, as compared to sham-treated, dams $(N=18,23$ respectively). d Specifically, the social preference ratio is significantly higher in the offspring of THC-treated dams (Two-tailed T-test, $P=$ 0.0130). e Both groups showed significant preference for the novel rat over the novel object (One-way ANOVA, $F_{3,78}=137.2 ;$ Tukey's post-hoc analysis, $P<0.0001$ for both groups). $\mathbf{f}$ The total time spent exploring the novel object and the novel rat did not differ between groups (Twotailed t-test, $P=0.1518) . \mathbf{g}-\mathbf{i}$ In the subsequent social memory test, the adult offspring of THC-treated dams failed to discriminate between the familiar and novel rats. $\mathbf{g}$ The social memory ratio is significantly higher in the offspring of sham-treated dams as compared to THC-treated dams (Two-tailed t-test, $P=0.0048)$. $\mathbf{h}$ The offspring of sham-treated dams showed a significant preference for the novel partner rat $(P<$ 0.0001 , Tukey's post-hoc analysis following a significant One-way ANOVA, $\left.F_{3,78}=9.016, P<0.0001\right)$. Conversely, no preference between the familiar and novel rats was demonstrated by the offspring of THC-treated dams $(P=0.9984$, Tukey's post-hoc analysis). i The total time spent exploring non-discriminated rats was significantly higher in the THC group (Two-tailed t-test, $P=0.0086$ ). ${ }^{*} P<0.05$.

inhibitor of Monoacyl-glycerol lipase the main enzyme degrading 2AG) in order to increase basal 2-AG levels prior to the 10-min, 10 $\mathrm{Hz}$ stimulation. Here, slices obtained from the adult offspring of THC-treated dams were found to exhibit robust, lasting LTD at layer 5 synapses following this pre-incubation. These data therefore indicate that increased levels of 2-AG in the PFC effectively restores eCB-LTD under these conditions.

Perinatal THC abolishes TBS-LTP in the PFC at adulthood Next, we elected to investigate additional forms of plasticity in the PFC of the adult offspring of THC-treated dams in order to determine the extent of altered PFC plasticity. Cannabinoids induced perturbations go beyond eCB-mediated synaptic plasticity [39, 40]. Notably, a single cannabinoid exposure abolishes theta-burst induced long-term potentiation (TBS-LTP) in the PFC of adult male rats [29]. In order to investigate whether a similar perturbation occurred in the PFC of adult, THC-exposed offspring, a TBS was applied to superficial layers of acute PFC slices obtained from these rats during simultaneous recording of extracellular field EPSPs at deep-layer synapses. Interestingly, while this TBS protocol effectively induced a lasting synaptic potentiation in slices obtained from the adult progeny of sham-treated dams, no such plasticity was observed in slices obtained from THC-treated dams (Fig. 3a, b). Sex-specific details can be found in Supplementary Table 4.

As with the loss of eCB-LTD, we sought to determine if increasing basal levels of endocannabinoids would mechanistically ameliorate this loss of LTP. Thus, slices were incubated with JZL184, for $>45$ minutes prior to recording. Here, the TBS protocol effectively elicited a strong and lasting synaptic potentiation (Fig. 3c, d). Thus, we observed that, as with the lost eCB-LTD, increased basal levels of 2-AG effectively restores lost TBS-LTP in the PFC of adult, THC-exposed rats.

Perinatal THC alters select parameters of cell-excitability in the PFC at adulthood

In utero cannabinoid exposure has previously been shown to alter basic parameters of cell-excitability in the PFC at adulthood [25]. Thus, we next performed patch-clamp recordings of deep-layer 
a

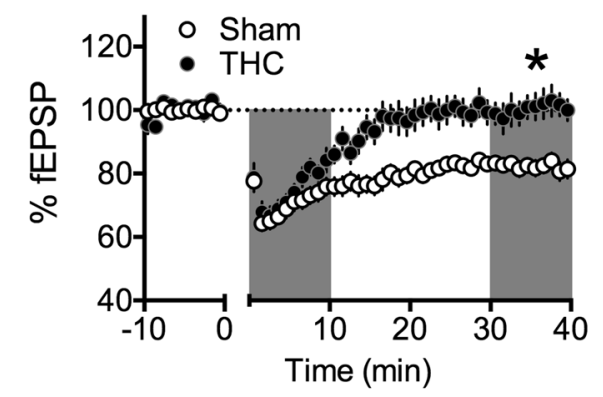

C

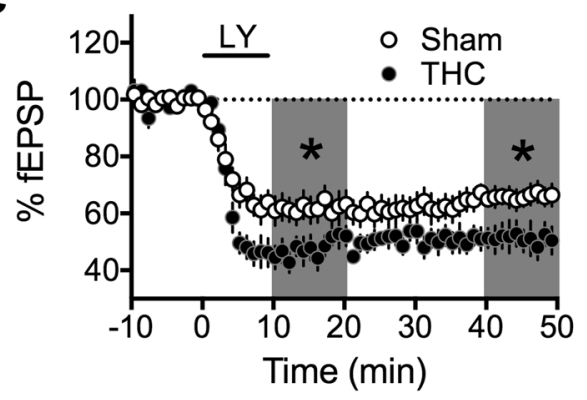

e

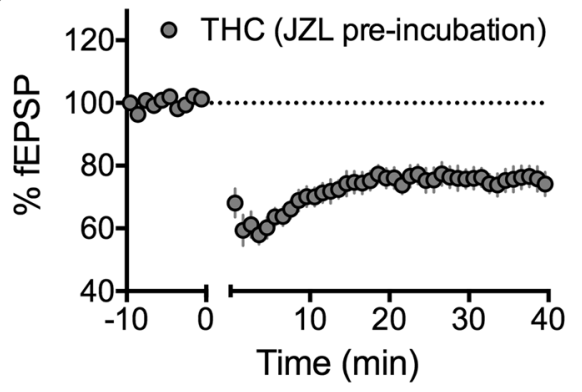

b
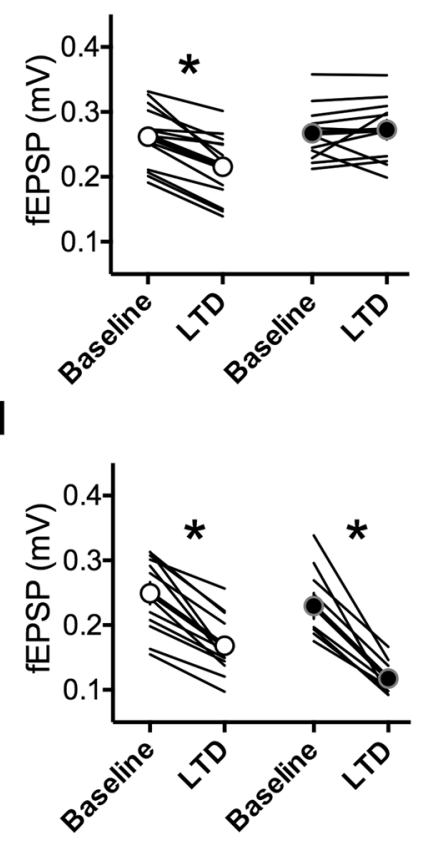

f

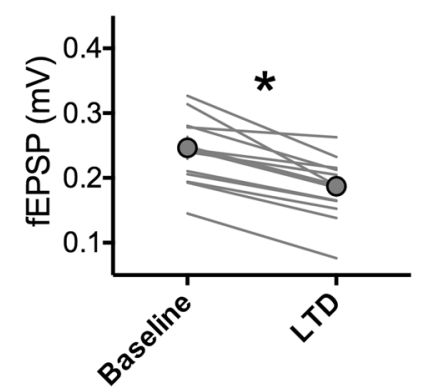

Fig. 2 Perinatal THC exposure induces a selective deficit in LTD in the PFC of adult offspring. a A 10-min, $10 \mathrm{~Hz}$ field stimulation of layer $2 / 3$ cells in the PFC of the adult offspring of vehicle-treated dams $(N=14)$ elicited a robust eCB-LTD at deep-layer synapses. However, this same protocol failed to induce eCB-LTD in the adult offspring of dams treated with THC $(N=11)$. No differences were found during the initial posttetanus period (0-10 min post-tetanus), however at 30-40 min post-tetanus, the baseline-normalized \% fEPSP was significantly lower in shamexposed, as compared to THC-exposed rats (Two-way RM ANOVA, $\mathrm{F}_{1,23}=10.63, P=0.0034$. Sidak's multiple comparisons test, $P=0.3972$ and $P<0.0001$ for $10 \mathrm{~min}$ and 40 min post-tetanus, respectively). b fEPSP magnitude at baseline ( $-10-0$ min) and LTD (30-40 min post-tetanus) values corresponding to the normalized values in a (Two-way RM ANOVA, $F_{1,23}=20.25, P=0.0002$. Sidak's multiple comparisons test, $P<$ 0.0001 and $P=0.7795$ for sham and THC, respectively). c: LTD mediated by mGlu $2 / 3$ receptors (mGluR-LTD) is augmented in THC-exposed offspring. mGluR-LTD, induced via a 10-min application of LY379268 (LY; $30 \mathrm{nM}$ ), produced a significant depression at deep-layer synapses of the PFC in the adult offspring of both sham- and THC-treated dams ( $N=12$ and 8 , respectively). During both the first ten-minutes post-drug and the last ten-minutes (i.e., 30-40 min post-drug), fEPSP depression was significantly larger in THC-exposed offspring, as compared to shamexposed (Two-way RM ANOVA, $\mathrm{F}_{1,18}=7.913, P=0.0120$. Sidak's multiple comparisons test, $P=0.0068$ and $P=0.0048$ at 10 -min and 40 -min, respectively). d fEPSP magnitude at baseline (-10-0 $\mathrm{min}$ ) and LTD (30-40 $\mathrm{min}$ post-drug) values corresponding to the normalized values in c (Two-way RM ANOVA, $F_{1,18}=105.5, P<0.0001$. Sidak's multiple comparisons test, $P<0.0001$ for both sham and THC). e, $\mathbf{f}$ Enhancing 2 -AG levels restores eCB-LTD in THC-exposed progeny. Following a > 45-min pre-incubation with the MAGL inhibitor, JZL 184 (4 $\mu$ M), the previously ineffective $10-\mathrm{min}, 10 \mathrm{~Hz}$ protocol effectively induced a significant eCB-LTD at deep-layer synapses of PFC slices obtained from the adult offspring of THC-treated dams $(N=11$; Two-tailed t-test, $P=0.0084) .{ }^{*} P<0.05$.

PFC pyramidal neurons in acute PFC slices obtained from the adult progeny of either sham- or THC-treated dams. An input-output curve revealed no significant differences between these groups (Fig. 4a), however a closer examination of current-injection induced action potentials revealed two key differences. First, the number of spikes elicited by current injections between 0-600 pA was significantly lower in THC-exposed offspring, as compared to sham controls (Fig. 4b). This effect was expectedly correlated with a significantly higher rheobase in these cells (Fig. 4c). However, no change in the resting membrane potential was noted (Fig. 4d), indicating that rather than being less excitable due to lower resting membrane values, other mechanisms drive the decreased excitability observed in these animals. Sex-specific details can be found in Supplementary Table 5.

\section{DISCUSSION}

Here, we have discovered that perinatal exposure to THC via lactation induces significant behavioral and electrophysiological alterations lasting into adulthood. Specifically, we found that this THC exposure 
a

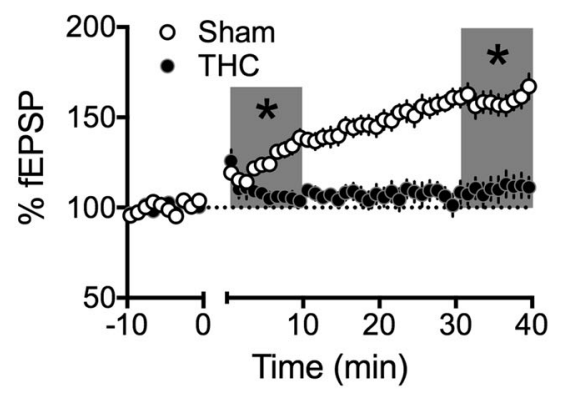

C

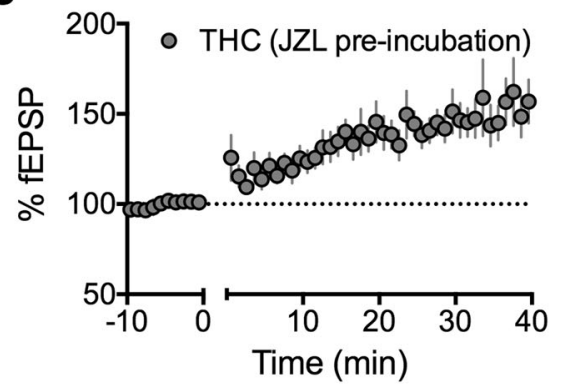

b

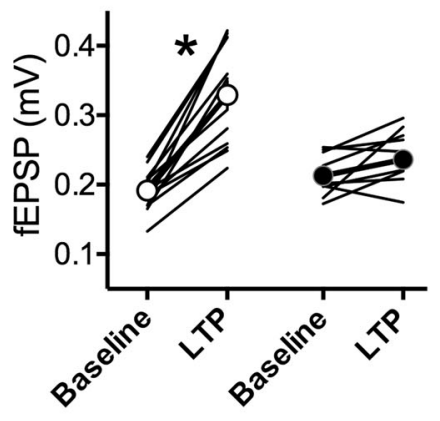

d

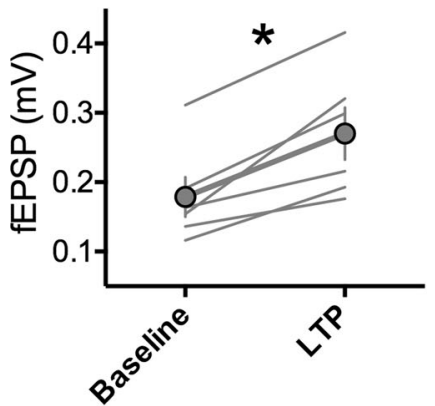

Fig. 3 Perinatal THC exposure abolishes theta-burst stimulation (TBS) LTP in the PFC of adult offspring in a 2-AG-dependent manner. a A TBS protocol (5 pulses at $100 \mathrm{hz}$, repeated 4 times) at layer $2 / 3$ cells in the PFC of the adult offspring of sham-treated dams $(N=13)$ elicited a robust LTP at deep-layer synapses. However, this same protocol failed to induce LTP in PFC slices obtained from the adult offspring of THCtreated dams $(N=10)$. During both the first $10 \mathrm{~min}$ post-tetanus and the last $10 \mathrm{~min}$ of recording (i.e., $30-40$ min post-tetanus), the normalized $\%$ fEPSP was significantly larger in slices obtained from sham-exposed rats, as compared to those of THC-exposed rats (Two-way RM ANOVA, $\mathrm{F}_{1,21}=32.57, P<0.0001$. Sidak's multiple comparisons test, $P=0.0094$ and $P<0.0001$ for 10 min and 40 min post-tetanus, respectively). $\mathbf{b}$ fEPSP magnitude at baseline $(-10-0 \mathrm{~min})$ and LTP (30-40 min post-tetanus) values corresponding to the normalized values in a (Two-way RM ANOVA, $\mathrm{F}_{1,21}=27.34, P<0.0001$. Sidak's multiple comparisons test, $P<0.0001$ and $P=0.3125$ for sham and THC, respectively). $\mathbf{c}$, $\mathbf{d}$ Enhancing 2-AG levels permits the subsequent induction of TBS-LTP in THC-exposed progeny. Following a $>45$-min pre-incubation with the MAGL inhibitor, JZL 184, the previously ineffective TBS protocol effectively induced a robust LTP at deep-layer synapses of PFC slices obtained from the adult offspring of THC-treated dams $(N=6$, Two-tailed T-test, $P=0.0085)$. ${ }^{*} P<0.05$.

alters social behavior, as well as synaptic plasticity and basic parameters of cell-excitability in the PFC of adult male and female offspring of dams given THC during the first 10 days of postnatal life.

First, our behavioral explorations revealed that perinatal THC exposure augments social exploration at the cost of discrimination between novel and social interactions. Interestingly, these results diverge from those found following in utero THC exposure, wherein social exploration has been found to be reduced $[25,41]$. Further, while in utero THC exposure appears to alter social behavior in a sexually divergent manner [25], here we have observed no differences between male and female progeny perinatally exposed to THC. The timing-specificity of long-term effects of drug exposure with regards to sexually dimorphic outcomes has been noted with several other substances, including tobacco [42], the anesthetic drug isoflurane [43] and toxins such as lead [44] and bisphenol-A [45].

While measurements of the total quantity of THC transferred to the developing offspring via breastmilk remains unknown in murine models, human data have calculated a transfer rate of $2.5 \%$ of the maternal dose to the developing infant via this route [7]. Further, analyses conducted on the breast milk of a daily consumer of cannabis in addition to analyses conducted on the blood plasma of the user's infant revealed detectable quantities of the metabolite $11-\mathrm{OH}-\mathrm{THC}$ in the infant, suggesting a sufficient quantity of THC was delivered via this route to produce detectable quantities of metabolized end-products in the developing offspring [8]. However, differences in volume of consumption between humans and rodents, as well as key differences in pharmacokinetic disposition of the drug between species suggests that future studies will be needed to determine the exact quantity of transferred THC in our regimen.

At a systems level, we observed multiple alterations to synaptic plasticity in the PFC. First, eCB-mediated LTD was entirely absent at deep-layer PFC synapses in slices obtained from the progeny of THC-treated dams. This finding is in line with previous findings from our lab demonstrating a loss of eCB-LTD in the PFC of in utero exposed offspring at adulthood [25] and extends upon previous findings that defects in PFC plasticity correlate with social dysfunction in other contexts, such as a mouse model of dietary polyunsaturated fat imbalance [32]. We surmise that this homeostatic compensation engages in order to maintain a functional working range of plasticity in the PFC and may result from increased availability of Gi/o messenger proteins which, prior to the loss of eCB-LTD, are likely to be sequestered by CB1 receptors [38] and therefore made less available for other forms of inhibitory plasticity, such as mGlu2/3-LTD.

The reappearance of eCB-LTD in the presence of enhanced 2AG levels achieved via pre-incubation with the potent and selective MAGL inhibitor, JZL184, extends and confirms previous similar findings in multiple models of environmental $[32,46,47]$ or genetic $[48,49]$ synaptopathies. The recovery of TBS-LTP following JZL184 pre-incubation may owe to a previously demonstrated relationship between presynaptic NMDA receptors and CB1R which have been shown to detect co-incident pre- and postsynaptic activation during certain forms of both LTD and LTP at deep-layer synapses of the PFC [47]. Moreover, in mouse models 
a

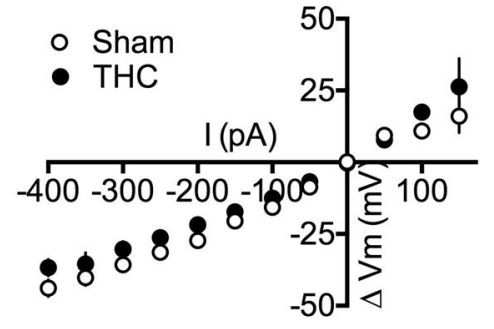

C

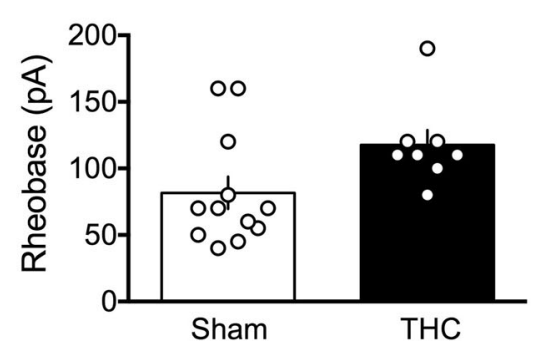

b

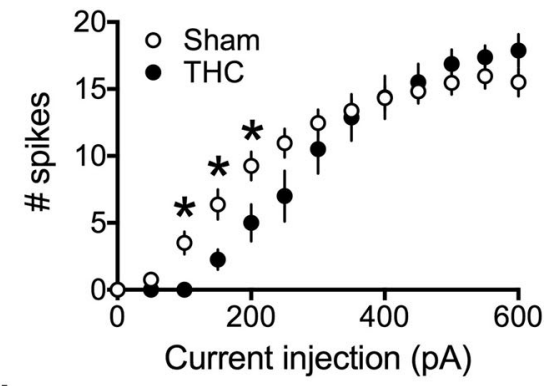

d

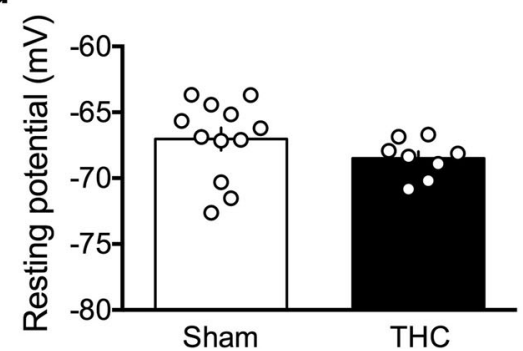

Fig. 4 Perinatal THC exposure alters properties of intrinsic excitability of deep-layer pyramidal neurons in the PFC of adult offspring. a Current injection steps of $50 \mathrm{pA}$ from $-400 \mathrm{pA}$ to $150 \mathrm{pA}$ revealed no differences in the I-V relationship in pyramidal neurons of the PFC between the adult offspring of sham- and THC-treated dams $(N=12,8$ respectively). b Action potentials elicited by progressive current injections from $0-600 \mathrm{pA}$ revealed a reduced number of spikes at low current injections in pyramidal neurons of the PFC in slices obtained from the adult offspring of THC-injected dams as compared to those from sham-treated dams. Significantly lower numbers of action potentials were observed following current injections of 100,150 and $200 \mathrm{pA}\left(N=8,12\right.$ respectively; Two-way RM ANOVA, $F_{20,360}=3.912, P<$ 0.0001 . Sidak's multiple comparisons test, $\left.{ }^{*} P<0.05\right)$. c: Progressive current injections in $10 \mathrm{pA}$ steps from $0-200 \mathrm{pA}$ revealed that the minimum current injection required to elicit an action potential (i.e., rheobase) was significantly higher in deep-layer pyramidal neurons of PFC slices obtained from the adult offspring of THC- as compared to sham-treated dams $(N=8,12$ respectively; Two-tailed t-test, $P=0.047)$. d No difference was observed in the resting membrane potential of deep-layer pyramidal cells in PFC slices obtained from the adult offspring of THC-treated dams, as compared to those obtained from sham-treated dams $(N=8,12$ respectively; Two-tailed t-test, $P=0.1646) .{ }^{*} P<0.05$.

of dietary-induced depression, deficient NMDAR-LTP at prefrontal [32] and hippocampal [47] synapses was rescued following JZL184 perfusion, further highlighting cross-talks between NMDA receptors and CB1R.

Finally, we observed that perinatal THC exposure decreases excitability of principle neurons of the PFC. As with other results of this study, this finding stands in contrast to those of earlier studies which have explored the consequences of in utero cannabinoid exposure, wherein increased excitability has been observed in the PFC [25]. Rather, this finding mirrors those of chronic exposure to THC later in development, such as during the adolescent period wherein decreased excitability of PFC neurons has been observed in mice [50]. Thus, we subscribe such differences to underlying state-dependent differences in the maturational window of development during which THC exposure takes place $[51,52]$.

Together, our results indicate that perinatal THC exposure via lactation induces lasting deficits at multiple scales which persist into adulthood. THC-exposed offspring exhibit increased social exploration at the cost of discrimination, coupled with significant alterations to multiple forms of plasticity in the PFC which are normalized via enhanced basal 2-AG ex vivo. Increased excitability of principal neurons of the PFC may underlie or accompany these issues, and further investigations are required to further characterize the extent to which basic synaptic transmission may be impacted by this early-life exposure. Further, both an increased breadth of behavioral investigations as well as extended characterizations of plasticity and synaptic functions in these animals in other brain regions are necessary to provide a more thorough picture of the extent to which perinatal cannabis exposure induces lasting deficits in brain function into adulthood.

\section{FUNDING AND DISCLOSURES}

This work was supported by the Institut National de la Santé et de la Recherche Médicale (INSERM); the INSERM-NIH exchange program (to AFS); Fondation pour la Recherche Médicale (Equipe FRM 2015 to OJJM) and the NIH (R01DA043982 to OJJM). The authors declare no competing interests.

\section{ACKNOWLEDGEMENTS}

The authors are grateful to the Chavis-Manzoni team members for helpful discussions.

\section{AUTHOR CONTRIBUTIONS}

AFS, MB, ALPA and OJJM designed research; AFS and MB performed research; AFS and $M B$ analyzed data; AFS, MB. and OJJM wrote the paper.

Publisher's note Springer Nature remains neutral with regard to jurisdictional claims in published maps and institutional affiliations.

\section{REFERENCES}

1. Azofeifa A, Mattson ME, Grant A. Monitoring marijuana use in the United States challenges in an evolving environment. JAMA - J Am Med Assoc. 2016;316:1765-6.

2. Fantasia HC. Pharmacologic implications of marijuana use during pregnancy. Nurs Womens Health. 2017;21:217-23.

3. Metz TD, Stickrath EH. Marijuana use in pregnancy and lactation: a review of the evidence. Am J Obstet Gynecol. 2015;213:761-78.

4. Young-Wolff KC, Sarovar V, Tucker LY, Conway A, Alexeeff S, Weisner C, et al. Selfreported daily, weekly, and monthly cannabis use among women before and during pregnancy. JAMA Netw Open. 2019;2(7):e196471. https://doi.org/10.1001/ jamanetworkopen.2019.6471. 
5. Ko JY, Farr SL, Tong VT, Creanga AA, Callaghan WM. Prevalence and patterns of marijuana use among pregnant and nonpregnant women of reproductive age. Am J Obstet Gynecol. 2015;213:201.e1-201.e10.

6. Dickson B, Mansfield C, Guiahi M, Allshouse AA, Borgelt LM, Sheeder J, et al. Recommendations from cannabis dispensaries about first-trimester cannabis use. Obstet. Gynecol. 2018;131:1031-8.

7. Baker T, Datta P, Rewers-Felkins K, Thompson H, Kallem RR, Hale TW. Transfer of inhaled cannabis into human breast milk. Obstet Gynecol. 2018;131:783-8.

8. Perez-Reyes M, Wall ME. Presence of delta9-tetrahydrocannabinol in human milk. N. Engl J Med. 1982;307:819-20.

9. Bertrand KA, Hanan NJ, Honerkamp-Smith G, Best BM, Chambers CD. Marijuana use by breastfeeding mothers and cannabinoid concentrations in breast milk. Pediatrics. 2018;142:e20181076. https://doi.org/10.1542/peds.2018-1076.

10. Laanterä S, Pietilä AM, Pölkki T. Knowledge of breastfeeding among pregnant mothers and fathers. J Perinat Neonatal Nurs. 2010;24:320-9.

11. Scheyer AF, Melis M, Trezza V, Manzoni OJJ. Consequences of perinatal cannabis exposure. Trends Neurosci. 2019:42:871-84.

12. Hurd YL, Manzoni OJ, Pletnikov MV, Lee FS, Bhattacharyya S, Melis M. Cannabis and the developing brain: insights into its long-lasting effects. J Neurosci 2019:39:8250-8.

13. Correa F, Wolfson ML, Valchi P, Aisemberg J, Franchi AM. Endocannabinoid system and pregnancy. Reproduction. 2016;152:R191-R200.

14. Taylor AH, Ang C, Bell SC, Konje JC. The role of the endocannabinoid system in gametogenesis, implantation and early pregnancy. Hum Reprod Update. 2007;13:501-13.

15. Fride E. Multiple roles for the endocannabinoid system during the earliest stages of life: pre- and oostnatal development. J Neuroendocrinol 2008;20:75-81.

16. Galve-Roperh I, Chiurchiù V, Díaz-Alonso J, Bari M, Guzmán M, Maccarrone $M$. Cannabinoid receptor signaling in progenitor/stem cell proliferation and differentiation. Prog Lipid Res. 2013;52:633-50.

17. Harkany T, Guzmán M, Galve-Roperh I, Berghuis P, Devi LA, Mackie K. The emerging functions of endocannabinoid signaling during CNS development. Trends Pharm Sci. 2007;28:83-92.

18. Harkany T, Mackie K, Doherty P. Wiring and firing neuronal networks: endocannabinoids take center stage. Curr Opin Neurobiol. 2008;18:338-45.

19. Jutras-Aswad D, DiNieri JA, Harkany T, Hurd YL. Neurobiological consequences of maternal cannabis on human fetal development and its neuropsychiatric outcome. Eur Arch Psychiatry Clin Neurosci. 2009;259:395-412.

20. Goldman-Rakic PS. Chapter 16 Cellular and circuit basis of working memory in prefrontal cortex of nonhuman primates. Prog Brain Res. 1991;85:325-36.

21. Seamans JK, Floresco SB, Phillips AG. Functional differences between the prelimbic and anterior cingulate regions of the rat prefrontal cortex. Behav Neurosci. 1995;109:1063-73.

22. Marsicano G, Lutz B. Expression of the cannabinoid receptor CB1 in distinct neuronal subpopulations in the adult mouse forebrain. Eur $J$ Neurosci. 1999;11:4213-25.

23. Scheyer AF, Martin HGS, Manzoni OJ The endocannabinoid system in prefrontal synaptopathies. In: Melis M. editor Endocannabinoids and Lipid Mediators in Brain Functions. Springer, Cham; 2017. https://doi.org/10.1007/9783-319-57371-7 7

24. Araque A, Castillo PE, Manzoni OJ, Tonini R. Synaptic functions of endocannabinoid signaling in health and disease. Neuropharmacology. 2017;124:13-24.

25. Bara A, Manduca A, Bernabeu A, Borsoi M, Serviado M, Lassalle O, et al. Sexdependent effects of in utero cannabinoid exposure on cortical function. ELife. 2018;77:e36234. https://doi.org/10.7554/eLife.36234.

26. Manduca A, Servadio M, Melancia F, Schiavi S, Manzoni O, Trezza V. Sex-specific behavioral deficits induced at early life by prenatal exposure to the cannabinoid receptor agonist WIN 55,212-2 depend on mGlu5 receptor signaling. Br J Pharmacol. 2020, https://doi.org/10.1111/bph.14879.

27. Scheyer AF, Borsoi M, Wager-Miller J, Pelissier-Alicot A-L, Murphy MN, Mackie K, et al. Cannabinoid Exposure via Lactation in Rats Disrupts Perinatal Programming of the Gamma-Aminobutyric Acid Trajectory and Select Early-Life Behaviors. Biol Psychiatry. 2020;87:666-7. https://doi.org/10.1016/j.biopsych.2019.08.023.

28. Martin HGS, Bernabeu A, Lassalle O, Bouille C, Beurrier C, Pelissier-Alicot AL, et al. Endocannabinoids mediate muscarinic acetylcholine receptor-dependent longterm depression in the adult medial prefrontal cortex. Front Cell Neurosci. 2015;9:457.

29. Borsoi M, Manduca A, Bara A, Lassalle O, Pelissier-Alicot AL, Manzoni OJ. Sex differences in the behavioral and synaptic consequences of a single In vivo exposure to the synthetic cannabimimetic win55,212-2 at puberty and adulthood. Front Behav Neurosci. 2019;13:23.
30. Kasanetz F, Lafourcade M, Deroche-Gamonet V, Revest JM, Berson N, Balado E, et al. Prefrontal synaptic markers of cocaine addiction-like behavior in rats. Mol Psychiatry. 2013;18:729-37.

31. Lafourcade M, Elezgarai I, Mato S, Bakiri Y, Grandes P, Manzoni OJ. Molecular components and functions of the endocannabinoid system in mouse prefrontal cortex. PLOS ONE. 2007;2:e709. https://doi.org/10.1371/journal.pone.0000709.

32. Manduca A, Bara A, Larrieu T, Lassalle O, Joffre C, Layé $S$, et al. Amplification of mGlu5-endocannabinoid signaling rescues behavioral and synaptic deficits in a mouse model of adolescent and adult dietary polyunsaturated fatty acid imbalance. J Neurosci. 2017;37:6851-68.

33. Van Kerkhof LW, Damsteegt R, Trezza V, Voorn P, Vanderschuren L. Social play behavior in adolescent rats is mediated by functional activity in medial prefrontal cortex and striatum. Neuropsychopharmacology. 2013;38:1899-909.

34. Otani S, Daniel H, Takita M, Crépel F. Long-term depression induced by postsynaptic group II metabotropic glutamate receptors linked to phospholipase C and intracellular calcium rises in rat prefrontal cortex. J Neurosci. 2002;22:3434-44.

35. Huang CC, Hsu Sen K. The role of NMDA receptors in regulating group II metabotropic glutamate receptor-mediated long-term depression in rat medial prefrontal cortex. Neuropharmacology. 2008;54:1071-8.

36. Huang CC, Yang PC, Lin HJ, Hsu Sen K. Repeated cocaine administration impairs group II metabotropic glutamate receptor-mediated long-term depression in rat medial prefrontal cortex. J Neurosci. 2007;27:2958-68.

37. Mato S, Robbe D, Puente N, Grandes P, Manzoni OJ. Presynaptic homeostatic plasticity rescues long-term depression after chronic Delta 9tetrahydrocannabinol exposure. J Neurosci J Soc Neurosci. 2005;25:11619-27.

38. Vásquez C, Lewis DL. The CB1 cannabinoid receptor can sequester G-proteins, making them unavailable to couple to other receptors. J Neurosci. 1999;19:9271-80.

39. Renard J, Vitalis T, Rame M, Krebs MO, Lenkei Z, Le Pen G, et al. Chronic cannabinoid exposure during adolescence leads to long-term structural and functional changes in the prefrontal cortex. Eur Neuropsychopharmacol. 2016;26:55-64.

40. Zlebnik NE, Cheer JF. Drug-induced alterations of endocannabinoid-mediated plasticity in brain reward regions. J Neurosci. 2016;36:10230-8.

41. Vargish GA, Pelkey KA, Yuan X, Chittajallu R, Collins D, Fang $C$, et al. Persistent inhibitory circuit defects and disrupted social behaviour following in utero exogenous cannabinoid exposure. Mol Psychiatry. 2017;22:56-67.

42. Cauley M, Hall BJ, Abreu-Villaça Y, Junaid S, White H, Kiany A, et al. Critical developmental periods for effects of low-level tobacco smoke exposure on behavioral performance. NeuroToxicology. 2018;68:81-7.

43. Sasaki Russell JM, Chinn GA, Maharjan D, Eichbaum Y, Sall JW. Female rats are more vulnerable to lasting cognitive impairment after isoflurane exposure on postnatal day 4 than 7. Br J Anaesth. 2019;122:490-9.

44. Anderson GD, Chan LN. Pharmacokinetic drug interactions with tobacco, cannabinoids and smoking cessation products. Clin Pharmacokinet. 2016;55:1353-68.

45. Rubin BS, Paranjpe M, DaFonte T, Schaeberle C, Soto AM, Obin M, et al. Perinatal BPA exposure alters body weight and composition in a dose specific and sex specific manner: The addition of peripubertal exposure exacerbates adverse effects in female mice. Reprod Toxicol. 2017;68:130-44.

46. Bosch-Bouju C, Larrieu T, Linders L, Manzoni OJ, Layé S. Endocannabinoidmediated plasticity in nucleus accumbens controls vulnerability to anxiety after social defeat stress. Cell Rep. 2016;16:1237-42.

47. Thomazeau A, Bosch-Bouju C, Manzoni O, Layé S. Nutritional n-3 PUFA deficiency abolishes endocannabinoid gating of hippocampal long-term potentiation. Cereb Cortex N Y N 1991. 2016. https://doi.org/10.1093/cercor/bhw052.

48. Jung K-M, Sepers $M$, Henstridge CM, Lassalle $O$, Neuhofer $D$, Martin $H$, et al. Uncoupling of the endocannabinoid signalling complex in a mouse model of fragile $X$ syndrome. Nat Commun. 2012;3:1080.

49. Thomazeau A, Lassalle O, lafrati J, Souchet B, Guedj F, Janel N, et al. Prefrontal deficits in a murine model overexpressing the down syndrome candidate gene dyrk1a. J Neurosci J Soc Neurosci. 2014;34:1138-47.

50. Pickel VM, Bourie F, Chan J, Mackie K, Lane DA, Wang G. Chronic adolescent exposure to $\Delta 9$-tetrahydrocannabinol decreases NMDA current and extrasynaptic plasmalemmal density of NMDA GluN1 subunits in the prelimbic cortex of adult male mice. Neuropsychopharmacology. 2019. https://doi.org/10.1038/s41386019-0466-9.

51. Campolongo P, Trezza V, Ratano P, Palmery M, Cuomo V. Developmental consequences of perinatal cannabis exposure: behavioral and neuroendocrine effects in adult rodents. Psychopharmacol (Berl). 2011;214:5-15.

52. Sjöström PJ, Turrigiano GG, Nelson SB. Neocortical LTD via coincident activation of presynaptic NMDA and cannabinoid receptors. Neuron. 2003;39:641-54. 